\title{
Avaliação da Variabilidade da Freqüência Cardíaca em Mulheres Climatéricas Treinadas e Sedentárias
}

\section{Evaluation of Heart Rate Variability in Trained and Sedentary Climacteric Women}

\author{
Mário Augusto Paschoal, Emily Assis Polessi, Fernanda Cardoso Simioni \\ Pontifícia Universidade Católica de Campinas - Campinas, SP - Brasil
}

\section{Resumo}

Fundamento: Alterações da função autonômica cardíaca são freqüentes no climatério e diferentes métodos têm sido empregados para conhecê-las e minimizá-las.

Objetivo: Estudar a interferência da atividade física dinâmica aeróbica de baixa intensidade sobre a variabilidade da freqüência cardíaca (VFC) de mulheres climatéricas.

Métodos: Estudo transversal que analisou a VFC de 15 mulheres climatéricas com média de idade de 56,8 \pm 4,9 anos, que já se encontravam em treinamento físico (caminhada de uma hora diária, três vezes por semana) há pelo menos dois anos (grupo ativo), e de 15 mulheres climatéricas $(56,5 \pm 3,7$ anos) sedentárias (grupo sedentário). Todas as voluntárias não faziam uso de reposição hormonal. Os dados da VFC foram comparados entre os grupos por meio do teste U de Mann-Whitney.

Resultados: Houve diferenças significativas tanto no domínio da freqüência como no domínio do tempo das seguintes variáveis da VFC, em medianas, para os grupos ativo e sedentário, respectivamente: potência total $\left(22.626,50 \mathrm{~ms}^{2}\right.$ e 4.432,10 ms $\mathrm{ms}^{2}$, componente baixa freqüêencia $\left(741,20 \mathrm{~ms}^{2}\right.$ e $\left.131,70 \mathrm{~ms}^{2}\right)$, componente alta freqüência $\left(668,90 \mathrm{~ms}^{2}\right.$ e $\left.131,70 \mathrm{~ms}^{2}\right)$, desvios padrão dos intervalos $\mathrm{RR}(51,60 \mathrm{~ms}$ e $22,50 \mathrm{~ms})$, raiz quadrada da soma dos quadrados das diferenças entre os intervalos RR $(35,30 \mathrm{~ms}$ e $15,90 \mathrm{~ms})$ e porcentagem de intervalos RR adjacentes maiores que $50 \mathrm{~ms}$ $(6,6 \%$ e $0,2 \%)$.

Conclusão: $\mathrm{O}$ estudo sugere que o treinamento aeróbio pode ter propiciado significativa melhoria da função autonômica cardíaca das mulheres climatéricas do grupo ativo, podendo ser uma opção útil para preservar essa condição funcional sem necessidade de terapias de reposição hormonal. (Arq Bras Cardiol 2008; 90(2): 80-86)

Palavras-chave: Climatério, mulheres, sistema nervoso autônomo, freqüência cardíaca.

\section{Summary}

Background: Changes in autonomic cardiac function are frequent during menopause, and various methods have been used to understand and minimize them

Objective: To study the interference of dynamic aerobic physical activity on heart rate variability (HRV) in climacteric women.

Methods: Cross-sectional study that analyzed HRV in 15 menopausal women (mean age $56.8 \pm 4.9$ years) who had participated in physical training (one-hour walks, 3 times a week) for at least two years (active group), and 15 menopausal women (mean age $56.5 \pm 3.7$ years) who were sedentary (sedentary group). None of the volunteers received hormonal replacement therapy. HRV data were compared between the groups by means of the Mann Whitney $U$ Test.

Results: There were significant differences both in the frequency and time domains of the following variables of HRV (in medians) for the active e sedentary groups, respectively: total power $\left(22,626.50 \mathrm{~ms}^{2}\right.$ and 4,432.10 ms 2 , low frequency component $\left(741.20 \mathrm{~ms}^{2}\right.$ and $\left.131.70 \mathrm{~ms}^{2}\right)$, high frequency component (668.90 $\mathrm{ms}^{2}$ and $\left.131.70 \mathrm{~ms}^{2}\right)$, standard deviations of RR intervals $(51.60 \mathrm{~ms}$ and $22.50 \mathrm{~ms})$, square root of the sum of squares of differences between the normal RR intervals $(35.30 \mathrm{~ms}$ and $15.90 \mathrm{~ms})$, and percentage of normal adjacent RR intervals greater than $50 \mathrm{~ms}(6.6 \%$ and $0.2 \%)$.

Conclusion: The study suggests that aerobic training may have afforded a significant improvement in the autonomic cardiac function of the menopausal women in the active group, and may be a useful option for preserving this functional condition without the need for hormonal replacement therapy. (Arq Bras Cardiol 2008;90(2):74-79)

Key words: Climacteric; women; autonomic nervous system; heart rate.

Correspondência: Mário Augusto Paschoal •

Rua Ferreira Penteado, 1242/72 - Cambuí - 13010-041 - Campinas, SP - Brasil

E-mail: fisioni@puc-campinas.edu.br

Artigo recebido em 17/04/07; revisado recebido em 18/06/07; aceito em 04/09/07. 


\section{Introdução}

O climatério é um período de diminuição fisiológica da função ovariana, durante o qual existem alterações endócrinas, somáticas e psíquicas. Pode ser definido como a fase do processo de envelhecimento que marca a transição da fase reprodutiva da vida para a fase pós-reprodutiva ${ }^{1}$.

Apesar de ser difícil determinar o momento exato de seu início, em geral o climatério começa em torno dos 40 anos de idade, variando consideravelmente de uma mulher para outra, com manifestações diversas. Sabe-se, porém, que as alterações hormonais e físicas estão em andamento e se dão de forma tão gradual que se tornam quase imperceptíveis ${ }^{2}$.

Com relação às doenças cardiovasculares (DCV), as mulheres apresentam os mesmos fatores de risco dos homens, ou seja, hipertensão arterial sistêmica, tabagismo, diabete melito, obesidade, vida sedentária, hipercolesterolemia, estresse e antecedentes familiares da enfermidade. No entanto, as DCV têm baixo índice durante os anos reprodutivos, em decorrência, principalmente, da manutenção dos níveis elevados de colesterol de lipoproteína de alta densidade (HDL-colesterol) por ação dos estrogênios. Após a menopausa, esse risco aumenta muito, aproximando-se progressivamente dos índices verificados nos homens ${ }^{2}$.

Dessa forma, a doença coronariana torna-se uma das principais causas de óbito no climatério, estando relacionada à diminuição do fluxo sangüíneo tecidual, ocasionado pela aceleração da formação de placas ateromatosas e pela alteração de mecanismos reguladores da vasomotricidade ${ }^{3}$. São responsáveis, respectivamente, por esses problemas as alterações do metabolismo das lipoproteínas ${ }^{4,5}$, dos carboidratos e da produção de insulina ${ }^{6}$, todos prováveis facilitadores do surgimento de obesidade ${ }^{7}$ e de alterações da pressão arterial $^{8}$, culminando com a piora do estado de hipoestrogenismo.

Dentre os vários métodos empregados para controlar o efeito das alterações funcionais cardíacas decorrentes do climatério, a atividade física tem se destacado, pelo seu amplo sucesso em oferecer benefícios cardíacos e multissistêmicos a seus praticantes, com ínfimos efeitos colaterais. A reposição hormonal, por seu lado, apesar de ter evoluído nesse aspecto, ainda traz certa preocupação pela sua relação com alguns tipos de neoplasias ${ }^{9}$ e o agravamento de quadros de miomas uterinos e a reativação de endometriose pélvica.

Entretanto, ainda são pouco freqüentes os estudos preocupados em analisar os benefícios da atividade física nessa população e, em especial, nas possíveis modificações adaptativas positivas das funções autonômicas cardíacas em resposta a programas de treinamento aeróbio.

No entanto, por ser uma ferramenta não-invasiva e de fácil acesso, a variabilidade da freqüência cardíaca (VFC) tem sido escolhida para esse tipo de investigação, pois, além de poder revelar as alterações cardíacas ligadas a programas de treinamento físico, é empregada em estudos sobre envelhecimento humano ${ }^{10-14}$, como o que se propôs na presente investigação.

Sendo assim, o objetivo deste estudo foi o de investigar a interferência da atividade física dinâmica aeróbica de baixa intensidade, realizada freqüentemente por mulheres climatéricas, em um marcador biológico ligado ao processo natural de envelhecimento representado pelo tônus e pela modulação da função autonômica cardíaca, extraídos da análise da VFC.

\section{Métodos}

Amostra - Fizeram parte da abordagem inicial para participação no estudo 37 mulheres na faixa etária de 45 a 65 anos, que estavam no período do climatério, clinicamente menopausadas e pós-menopausadas. De acordo com os critérios de inclusão e exclusão, as mulheres foram divididas em dois grupos, tendo sido 7 delas excluídas do estudo em decorrência de achados irregulares em seus traçados eletrocardiográficos, sugerindo a presença de afecções. Essas mulheres foram orientadas a procurar atendimento médico especializado.

As 30 mulheres restantes compuseram os dois grupos do estudo, ativo e sedentário, com 15 mulheres em cada grupo. As 15 mulheres escolhidas para compor o grupo ativo tinham média de idade de 56,8 \pm 4,9 anos e participavam, há pelo menos dois anos, do grupo de mulheres da terceira idade proporcionado pela Secretaria de Ação Social de um município do Estado de São Paulo. As 15 mulheres escolhidas para compor o grupo sedentário tinham média de idade de $56,5 \pm 3,7$ anos.

Critérios de inclusão para o grupo das sedentárias - Poderiam participar desse grupo as mulheres que clinicamente comprovassem estar no período de climatério (amenorréia há pelo menos um ano), que fossem sedentárias, que não praticassem nenhum tipo de atividade física regularmente há pelo menos seis meses, que não executassem trabalho caseiro de grande intensidade (como a realização de faxinas), que não fossem tabagistas, que não estivessem fazendo uso de medicação que pudesse interferir nos resultados, que não realizassem terapia de reposição hormonal, e que não apresentassem história de doenças cardiovasculares e metabólicas.

Critérios de inclusão para o grupo das ativas - Os mesmos pré-requisitos definidos para o grupo das sedentárias, porém deveriam estar praticando, há pelo menos dois anos, exercício físico aeróbio leve a moderado (caminhada pelo menos três vezes por semana, com sessões de 50 a 60 minutos), e a freqüência cardíaca $(\mathrm{FC})$ deveria ser mantida numa zona entre 100 batimentos por minuto (bpm) e 110 bpm nos primeiros três meses e nos meses subseqüentes, entre 110 bpm e 120 bpm.

Critérios de exclusão do estudo - Não poderiam fazer parte do estudo aquelas mulheres que não preenchessem os critérios de inclusão e que, por qualquer motivo, se negassem a assinar o termo de consentimento livre e esclarecido.

O projeto de pesquisa foi aprovado por um Comitê de Ética em Pesquisas Envolvendo a Participação de Seres Humanos.

\section{Procedimentos}

Os procedimentos se iniciaram no dia anterior à coleta de dados, com explicações a todas as participantes de que 24 horas antes dos exames não poderiam se exercitar nem ingerir bebidas alcoólicas, café, chá, chocolate e refrigerantes 


\section{Artigo Original}

com estimulantes, e que deveriam ter uma noite de sono de, no mínimo, sete horas.

A coleta de dados foi feita sempre pela manhã, com aferição do peso e da estatura corporais (balança Filizolla ${ }^{\circledR}$ ) e, também, dos dados clínicos, conforme apresentado na tabela 1.

Em seguida, para que se procedesse ao registro dos batimentos cardíacos para posterior análise da VFC, todas as voluntárias foram conduzidas a uma sala com temperatura controlada a $23^{\circ} \mathrm{C}$, em ambiente silencioso, e tiveram um cardiofreqüencímetro (modelo S810 Heart Rate Monitor ${ }^{\circledR}$, Kempele - Finlândia) fixado a seu tórax. Depois, foram posicionadas em supino e assim permaneceram por 12 minutos, tempo total do registro. Durante essa ação, as voluntárias foram orientadas a não conversar e nem se mover só podendo fechar os olhos para obter melhor relaxamento.

Posteriormente, os batimentos registrados foram direcionados a um computador por meio de uma interface IR-Polar ${ }^{\circledR}$ de emissão de sinais infravermelhos, sendo usados os programas Polar Precision Performance ${ }^{\circledR}$ (Finlândia) e Nevrokard HRV ${ }^{\circledR}$ (Eslovênia) para análise da VFC feita no domínio do tempo (DT) e no domínio da freqüência (DF).

A análise da VFC no DT se refere à aplicação de determinados procedimentos estatísticos sobre sucessivos intervalos RR (iRR) normais do eletrocardiograma (ECG), delimitados num determinado período ${ }^{13}$. Sendo assim, podem, por exemplo, ser calculados a média dos iRR e o desvio padrão da média dos $i R R$, entre outras possibilidades, cada uma delas com um significado representativo da função autonômica, refletindo o balanço vago-simpático cardíaco do momento.

A análise no DF, também designada como análise espectral, consiste em método de estudo que permite decompor a VFC de determinado período em seus componentes oscilatórios fundamentais, definindo-os pela sua freqüência e amplitude ${ }^{15}$.

Para a análise da VFC, foram eliminados o primeiro e o último minutos do registro, separando-se os 10 minutos centrais, período esse, de maior estabilidade do traçado. Além disso, para garantia da qualidade do sinal e eliminação

Tabela 1 - Valores de idades, antropométricos e clínicos das voluntárias

\begin{tabular}{lccc}
\hline Variáveis & $\begin{array}{c}\text { Grupo ativo } \\
\text { (média } \pm \text { desvio } \\
\text { padrão) }\end{array}$ & $\begin{array}{c}\text { Grupo sedentário } \\
\text { (média } \pm \text { desvio } \\
\text { padrão) }\end{array}$ & $\begin{array}{c}\text { Valor } \\
\text { de p }\end{array}$ \\
\hline Idade (anos) & $56,8 \pm 4,9$ & $56,5 \pm 3,7$ & 0,91 \\
Peso $(\mathrm{kg})$ & $69,4 \pm 10,4$ & $74,2 \pm 13,5$ & 0,45 \\
Estatura (cm) & $160,0 \pm 0,0$ & $161,0 \pm 2,0$ & 1,0 \\
IMC & $26,6 \pm 3,5$ & $28,2 \pm 3,8$ & 0,38 \\
Freqüência & $64,1 \pm 8,6$ & $70,2 \pm 8,9$ & 0,08 \\
cardiaca (bpm) & $125,0 \pm 8,6$ & $125,3 \pm 11,2$ & 0,86 \\
Pressão arterial & & & \\
sistólica (mmHg) & $82,3 \pm 6,7$ & $80,6 \pm 9,6$ & 0,39 \\
$\begin{array}{l}\text { Pressão arterial } \\
\text { diastólica (mmHg) }\end{array}$ & & & \\
\hline IMC índice de massa & & &
\end{tabular}

IMC - índice de massa corporal. de prováveis artefatos, foi aplicado um filtro muito forte, padrão dos próprios programas, por meio dos quais foram obtidos relatórios com os valores das variáveis do DT e do DF, submetidos a tratamento estatístico.

Para a avaliação da VFC no DT, foram analisados os valores dos iRR médios normais, os desvios padrão dos iRR médios normais, a raiz quadrada da média da soma dos quadrados das diferenças entre iRR adjacentes (RMSSD), e a porcentagem de iRR adjacentes cuja diferença fosse maior que $50 \mathrm{~ms}$ (pNN50).

Com relação ao DF, foram estudadas as potências totais (PT, variância dos iRR normais sobre o segmento temporal do registro $^{13}$ ), os valores dos componentes de baixa freqüência ( $\mathrm{BF}$, mais relacionados à atividade simpática cardíaca) e de alta freqüência ( $A F$, relativos à atividade parassimpática cardíaca), em valores absolutos, além da razão BF/AF.

\section{Tratamento estatístico}

Para as análises comparativas das variáveis da VFC entre os grupos, o teste utilizado foi o teste $U$ de Mann-Whitney. Os resultados estão apresentados em caixas de valores (boxplots), sendo destacados os valores de medianas, 1ㅇ e 3ㅇ quartis, e os valores extremos. A escolha recaiu sobre esse teste, pois o estudo em pauta trata de dados cujo interesse é conhecer as diferenças entre dois grupos (ativo e sedentário). Os procedimentos de medição/avaliação dão conhecimento de dados com nível de mensuração no mínimo ordinal.

Por nível de mensuração ordinal deve-se entender que é conhecida a hierarquia dos dados (por postos), mas a intensidade e o grau das diferenças não são totalmente conhecidos ou não são perfeitamente exatos. $O$ teste $U$, por ser não-paramétrico, é apropriado ao caso presente, por envolver duas amostras distintas (independentes) submetidas ao mesmo procedimento de coleta de dados.

Pela mesma razão, pode-se, também, classificar as amostras como sendo não-pareadas. Além disso, o teste $U$ de MannWhitney é adequado à avaliação de amostras pequenas e médias, como as do presente estudo, permitindo julgamentos de boa qualidade. Dessa forma, apesar do número pequeno de voluntárias, os resultados dos testes aplicados corresponderam a $p \leq 0,01$ entre os valores das variáveis, significando que as diferenças verificadas nas amostras podem ser estendidas à população.

\section{Resultados}

Com relação às idades e às avaliações antropométricas e clínicas (tab. 1), pode-se perceber que os grupos são homogêneos, não ocorrendo diferença significativa entre os dados comparados, não interferindo, portanto, no estudo.

Com relação aos registros dos batimentos cardíacos, a análise da VFC realizada no DF pode ser visualizada nas figuras 1, 2 e 3, com a apresentação dos boxplots. Ocorreram diferenças significativas ( $p<0,0001$ ) entre ambos os grupos, considerando os valores das variáveis $\mathrm{PT}, \mathrm{BF}$ e AF absolutos. $\mathrm{O}$ grupo ativo apresentou valores de medianas para PT, AF e BF, respectivamente, de 22.626,5 $\mathrm{ms}^{2}, 741,2 \mathrm{~ms}^{2}$ e $668,9 \mathrm{~ms}^{2}$. O grupo sedentário apresentou, em medianas, para PT, AF e BF, 


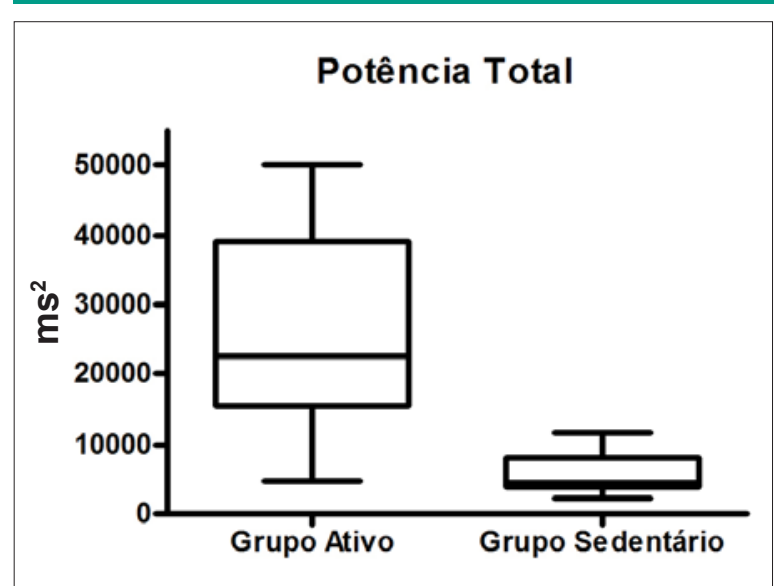

Fig. 1 - Valores de potência total obtidos nos grupos ativo e sedentário, por meio da análise da variabilidade da freqüência cardíaca (VFC) feita no domínio da freqüência (DF). Os dados estão apresentados em forma de boxplots, que representam os valores de medianas, $1^{\circ}$ e $3^{\circ}$ quartis e valores extremos.

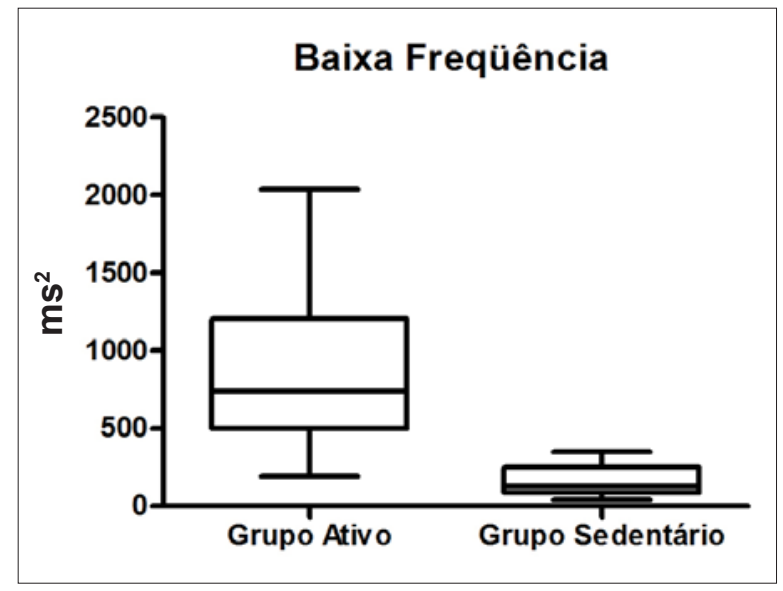

Fig. 2 - Valores de baixa freqüência obtidos nos grupos ativo e sedentário, por meio da análise da variabilidade da freqüência cardíaca (VFC) feita no domínio da freqüência (DF). Os dados estão apresentados em forma de boxplots, que representam os valores de medianas, $1^{\circ}$ e $3^{\circ}$ quartis e valores extremos.

respectivamente, 4.431,1 ms ${ }^{2}, 131,7 \mathrm{~ms}^{2}$ e 119,4 $\mathrm{ms}^{2}$.

Esses dados demonstram que tanto o tônus simpático como o tônus parassimpático foram maiores no grupo de mulheres ativas, tendo, ambos, importante influência no valor da PT.

Em contrapartida, os valores das razões BF/AF, por levar em conta para seu cálculo os valores de BF e AF em unidades normalizadas, não foram diferentes entre os grupos.

Apesar de os valores dos iRR não se mostrarem diferentes na análise da VFC no DT, pode-se constatar, observando-se as figuras 4, 5 e 6, que houve significativa diferença entre os valores de medianas dos grupos para: a) desvios padrão dos iRR, com 51,6 ms para o grupo ativo e 22,5 ms para o grupo sedentário; b) RMSSD, com 35,3 ms e 15,9 ms para os grupos ativo e sedentário, respectivamente; e c) pNN50, com o grupo ativo apresentando 6,6\% e o grupo sedentário, 0,2\%.

Essas diferenças expressam modificações cardíacas

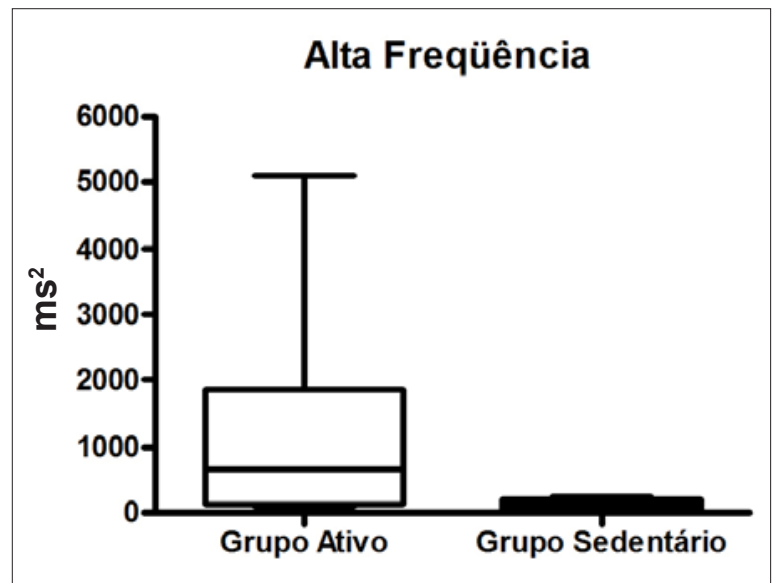

Fig. 3 - Valores de alta freqüência obtidos nos grupos ativo e sedentário, por meio da análise da variabilidade da freqüência cardíaca (VFC) feita no domínio da freqüência (DF). Os dados estão apresentados em forma de boxplots, que representam os valores de medianas, $1^{\circ} \mathrm{e} 3^{0}$ quartis e valores extremos.

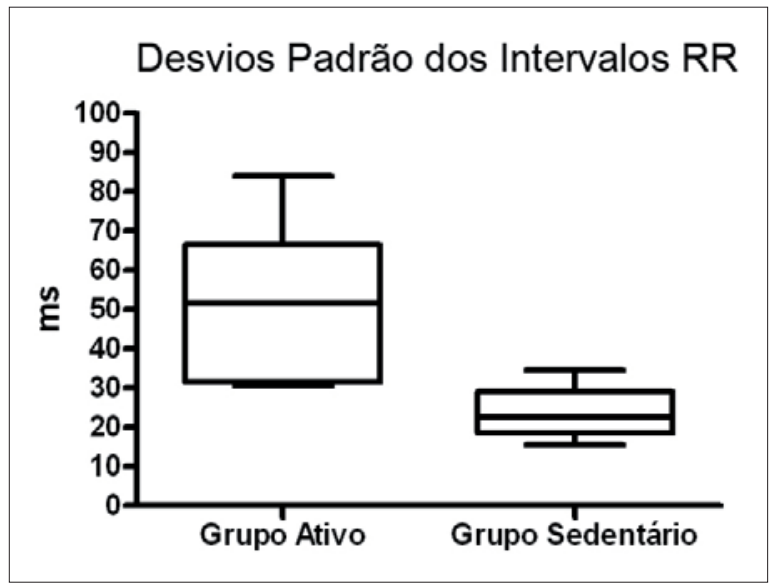

Fig. 4 - Valores dos desvios padrão dos intervalos RR médios obtidos nos grupos ativo e sedentário, por meio da análise da variabilidade da freqüência cardíaca (VFC) feita no domínio do tempo (DT). Os dados estão apresentados em forma de boxplots, que representam os valores de medianas, $1^{\circ}$ e $3^{\circ}$ quartis e valores extremos.

autonômicas importantes presentes no grupo ativo, captadas na análise feita no DT e no DF, as quais, de forma geral, refletiram a maior sensitividade autonômica por parte desse grupo.

Igualmente, cabe ressaltar que todas essas alterações positivas referentes à modulação autonômica cardíaca estão ligadas à arritmia sinusal respiratória ${ }^{16}$ (alterações cíclicas dos batimentos cardíacos em sintonia com o ato respiratório), muito mais presentes em jovens ${ }^{13}$, atletas ${ }^{17} \mathrm{e}$ em pessoas que têm hábitos saudáveis.

\section{Discussão}

Apesar de existir uma tendência natural de ganho de peso em pessoas sedentárias ${ }^{18}$, e por não se ter feito controle alimentar das participantes do estudo, houve a preocupação de o peso corporal das voluntárias ser similar, evitando 


\section{Artigo Original}

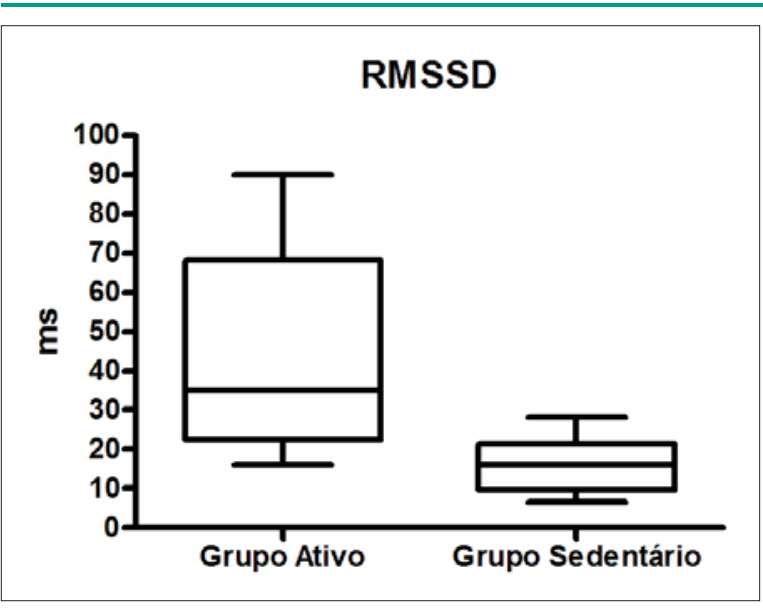

Fig. 5 - Valores das raízes quadradas das somas dos quadrados das diferenças dos intervalos RR (RMSSD) obtidos nos grupos ativo e sedentário, por meio da análise da variabilidade da freqüência cardíaca (VFC) feita no domínio do tempo (DT). Os dados estão apresentados em forma de boxplots, que representam os valores de medianas, $1^{\circ}$ e $3^{\circ}$ quartis e valores extremos.

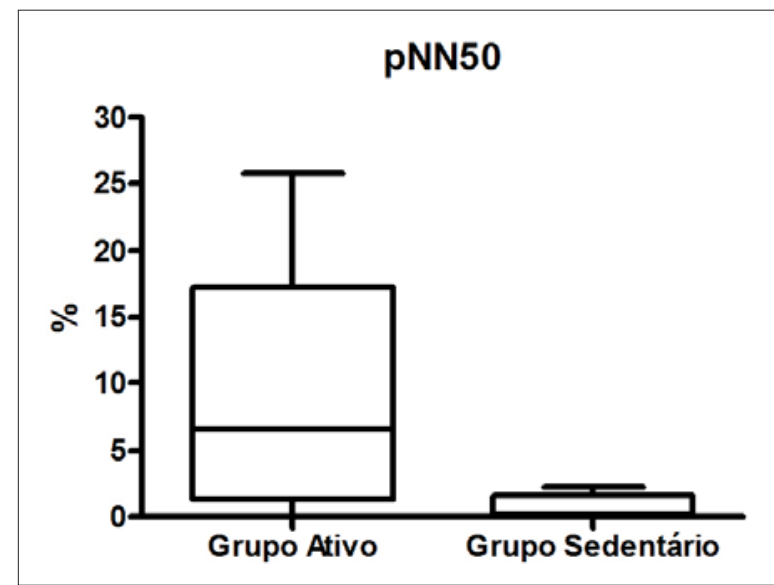

Fig. 6 - Valores das porcentagens de intervalos RR adjacentes maiores que 50 ms (pNN50) obtidos nos grupos ativo e sedentário, por meio da análise da variabilidade da freqüência cardíaca (VFC) feita no domínio do tempo (DT). Os dados estão apresentados em forma de boxplots, que representam os valores de medianas, $1^{\circ}$ e $3^{\circ}$ quartis e valores extremos.

interferência nos resultados. Dessa forma, pode-se notar que os valores dessa variável e de todas as demais apresentadas na tabela 1 são muito parecidos entre os grupos, conferindo maior padronização da amostra nesses aspectos.

Entretanto, com relação à FC de repouso, os estudos demonstram tendência à redução dos valores dessa variável nas pessoas treinadas, sendo diferentes dos valores de sedentários ${ }^{19}$. No presente estudo, porém, apesar de esse fato não ter ocorrido, o que talvez se deva ao número de voluntários (já que o valor de p foi de 0,08 , muito próximo da significância), os valores de FC encontrados não influenciaram a análise da VFC, que mostrou significativa diferença da modulação autonômica entre os grupos.

O que se constatou é que os valores de FC documentados ao repouso e incluídos na tabela 1 foram fiéis aos valores dos
iRR médios registrados por 10 minutos e que serviram de base para o cálculo da VFC. Conforme pode ser observado, os valores desses intervalos não diferiram entre os grupos durante a análise da VFC.

Quanto aos demais dados apresentados na tabela 1, cabe ressaltar que essa similaridade entre eles se deve ao fato de o grupo sedentário ser constituído de mulheres saudáveis, portanto com os valores das variáveis apresentando-se dentro dos parâmetros de normalidade.

A VFC foi utilizada para se determinar prováveis diferenças da modulação autonômica cardíaca entre as mulheres dos respectivos grupos. A intenção de se utilizar essa ferramenta decorre do fato de a mesma poder oferecer, sob condições controle, subsídios de extrema sensibilidade (sintonia fina) dos mecanismos de ajustes cardíacos, batimento a batimento, em resposta às necessidades homeostáticas e teciduais ${ }^{20,21}$ e, também, por já ter sido empregada em outros estudos envolvendo mulheres no climatério ${ }^{22,23}$.

Sabe-se que as atividades eferentes vagal e simpática direcionadas ao nódulo sinoatrial, caracterizadas por descarga altamente sincronizada com cada ciclo cardíaco, podem ser moduladas por componentes oscilatórios centrais (centros vasomotor e respiratório) e periféricos (oscilações da pressão arterial e movimentos respiratórios) ${ }^{16,24}$ e todos esses componentes oscilatórios estão presentes nas condições controle utilizadas na presente investigação, gerando flutuações rítmicas nas descargas nervosas eferentes, que se manifestam, no coração, por oscilações de curta e longa duração dos iRR, conferindo maior ou menor VFC e, portanto, maior ou menor tônus autonômico cardíaco ${ }^{13}$.

Entretanto, com o avanço da idade e a queda da produção de estrogênio, observa-se a redução do componente parassimpático e, conseqüentemente, da VFC nas mulheres após a menopausa ${ }^{25}$.

Esse aspecto pode ser ressaltado no estudo de Brockbank e cols. ${ }^{25}$, que constataram redução estatisticamente significante do iRR das mulheres pós-menopausadas, o que reforça cada vez mais as evidências de diminuição da contratilidade cardíaca $^{26}$, de declínio da sístole ventricular esquerda ${ }^{27}$ e de alterações dos barorreceptores no período pós-menopausa.

Mais relevante que os próprios iRR, os desvios padrão dos iRR médios refletem mais apropriadamente a modulação vagal sobre o coração $0^{11,13}$, pois quanto maior o desvio padrão dos iRR encontrado maior será o tônus parassimpático. Exatamente como o constatado no presente estudo, com significativa diferença dos desvios padrão dos iRR entre os grupos, com maiores valores apresentados pelas mulheres ativas.

Dessa forma, credita-se aos exercícios aeróbios essa modificação positiva à saúde cardiovascular desse grupo de mulheres, tal qual já fora documentado em outros estudos ${ }^{11,28-32}$ envolvendo pessoas dessa mesma faixa etária.

Myslivecek e cols. ${ }^{28}$ apresentaram resultados similares aos deste estudo ao pesquisarem se o treinamento aeróbio moderado influenciava a VFC e a função espontânea barorreflexa em mulheres de meia-idade. Para isso, foram selecionadas 32 mulheres sedentárias, incluindo pré e pósmenopausadas. Após o treinamento, os resultados sugeriram que mesmo um curto programa de exercícios aeróbios, bem 
consistente e de acordo com as diretrizes para a atividade física proposta, pode aumentar a modulação vagal em mulheres de meia-idade.

Perini e cols. ${ }^{29}$ também demonstraram aumento da modulação autonômica cardíaca, constatado pelas análises da VFC feitas antes e após programa de treinamento aeróbio moderado a vigoroso, aplicado em homens e mulheres idosos. Além disso, como também foi documentado no presente estudo, esses autores relataram maiores valores de PT no grupo treinado.

Em 2004, Jurca e cols. ${ }^{33}$ realizaram um estudo randomizado controlado com 88 mulheres pós-menopausadas sedentárias divididas em dois grupos. Desse total, 49 participaram de um programa de exercício aeróbio com duração de oito semanas, tendo em média 44 minutos cada sessão, realizado três a quatro vezes por semana, com intensidade de $50 \%$ do $\mathrm{VO}_{2}$ máx; as outras 39, por sua vez, formaram o grupo controle, que continuou sua vida normal. O estudo demonstrou que, após as oito semanas, as mulheres que participaram do treinamento apresentaram diminuição da FC de repouso, além de aumento de todos os valores das variáveis no DT e no DF da VFC, principalmente RMSSD, BF, AF e PT em unidades absolutas.

Como no presente estudo, a intensidade dos exercícios aplicada por Jurca e cols. ${ }^{33}$ foi leve, e os resultados dos valores das variáveis no DF, em unidades normalizadas, também não apresentaram mudanças após o treinamento.

Às vezes a simples análise da VFC em unidades normalizadas (u.n.) sem a devida atenção pode causar a falsa impressão de que não houve diferença entre os dados analisados. Mas cabe a ressalva de que os $62,5 \%$ (em u.n.) de BF das mulheres ativas significam, em valores absolutos, $741,2 \mathrm{~ms}^{2}$; para as sedentárias, seus 62,1\% de BF significam apenas $131,7 \mathrm{~ms}^{2}$; os $37,5 \%$ de AF das ativas representam, em valores absolutos, $668,9 \mathrm{~ms}^{2}$; e os $37,9 \%$ das sedentárias equivalem a $119,4 \mathrm{~ms}^{2}$, com significativa diferença entre os valores.

As limitações deste estudo foram: o número de mulheres avaliadas, a ausência do controle de fatores estressantes que poderiam interferir nos resultados, além da ausência de exames de função tireoidiana (TSH, T4), creditando-se a higidez das voluntárias apenas a seus parâmetros clínicos.

Com relação a futuros estudos, acreditamos que ainda há muito a ser investigado sobre os efeitos das diferentes intensidades e durações dos exercícios aeróbios. No entanto, já existem fortes indicativos de que o que importa é realizar a atividade física, pois tanto nos estudos cuja intensidade da

\section{Referências}

1. Copeland JF. Tratado de ginecologia. Rio de Janeiro: Guanabara Koogan; 1996.

2. Fernandes CE. Menopausa: diagnóstico e tratamento. São Paulo: Editora Solvay Farma; 2003.

3. Satmpfer MJ, Colditz GA. Estrogen replacement therapy and coronary heart atividade foi leve como naqueles nos quais ela foi moderada todos demonstraram resultados animadores sobre a positiva modificação da VFC.

\section{Conclusões}

Concluiu-se que a prática freqüente de exercícios físicos aeróbios leves, com a FC controlada entre 100 bpm e 120 bpm, realizados três vezes por semana, por dois anos, em mulheres no climatério sem reposição hormonal pode ter sido responsável pela melhora da função autonômica cardíaca revelada pela análise da VFC, representada pelo aumento significativo da modulação vagal e simpática no DF, com reflexos importantes no valor da PT.

Da mesma forma, no DT, a análise da VFC demonstrou significativa melhoria dos componentes que refletem a atividade vagal das mulheres ativas, considerada protetora do coração, confirmando assim que essa análise pode ser um parâmetro muito importante nesse tipo de investigação.

Dessa forma, a atividade física aeróbia freqüente, mesmo de baixa intensidade, demonstrou ser excelente opção à preservação e/ou à melhoria da função autonômica cardíaca para as mulheres climatéricas que não toleram o uso de terapia de reposição hormonal, seja por qualquer tipo de desconforto ou mesmo por apresentarem receio da possibilidade, ainda discutível, de esse tipo de terapêutica ser possível causadora de neoplasias, agravamento de miomas uterinos e reativação de endometriose pélvica, além do risco para o desenvolvimento de doenças tromboembólicas.

\section{Agradecimento}

Conselho Nacional de Desenvolvimento Científico e Tecnológico (CNPq).

\section{Potencial Conflito de Interesses}

Declaro não haver conflito de interesses pertinentes.

\section{Fontes de Financiamento}

O presente estudo foi parcialmente financiado pelo Conselho Nacional de Desenvolvimento Científico e Tecnológico (CNPq).

\section{Vinculação Acadêmica}

Não há vinculação deste estudo a programas de pósgraduação. disease: a quantitative assessment of the epidemiologic evidence. Prevent Med. 1991; 20: 47-63.

4. Henderson BE, Paganini-Hill A, Ross PK. Decreased mortality in users of estrogen replacement therapy. Arch Intern Med. 1991; 151-7.

5. Stevenson JC, Crook D, Godsland IF, Collins P, Whitehead MI. Hormone 


\section{Artigo Original}

replacement therapy and cardiovascular system nonlipid effects. Drugs. 1994; $47: 35-41$

6. Walton C, Godsland IF, Proudler AJ, Wynn V, Stevenson JC. The effects of the menopause on insulin sensitivity, secretion and elimination in non-obese, healthy women. Eur J Clin Invest. 1993; 23: 466-73.

7. Haarbo J, Marslew U, Gotfredsen A, Christiansen C. Postmenopausa hormone replacement therapy prevents central distribution of body fat after menopause. Metabolism. 1991; 40: 1323-6.

8. Brosnihan KB, Moriguchi A, Nakamoto H, Dean RH, Ganten D, Ferrario $\mathrm{CM}$. Estrogen augments the contribution of nitric oxide to blood pressure regulation in transgenic hypertensive rats expressing the mouse Ren-2 gene. Am J Hypertens. 1994; 7: 576-82.

9. Halbe HW. Tratado de ginecologia. 2ae ed, São Paulo: Ed. Roca, 1993.

10. Agelink MW. Standardized tests of heart rate variability: normal ranges obtained from 309 heathy humans, and effects of age, gender and heart rate. Clin Auton Res. 2001; (11): 99-108.

11. Paschoal MA, Volanti VM, Fernandes FC. Variabilidade da freqüência cardíaca em diferentes faixas etárias. Rev Bras Fisioter. 2006; 10 (4): 4139.

12. Silva CS, Marques LS, Moraes FR, Catai AM, Oliveira L, Silva E. Investigação da variabilidade da freqüência cardíaca de mulheres nos períodos manhã e noite. Rev Bras Fisioter. 2001; 5 (2):65-71.

13. Heart rate variability: standards of measurement physiological interpretation and clinical use. Task Force of the European Society of Cardiology and the North American Society of Pacing and Electrophysiology. Circulation. 1996; 93 (5):1043-65.

14. Migliaro ER, Contreras P, Bech S, Etxagibel A, Castro M, Ricca R, et al. Relative influence of age, resting heart rate and sedentary life style in short term analysis of heart rate variability. Braz J Med Biol Res. 2001; 34 (4): 493-500.

15. Longo A, Ferreira D, Correia MJ. Variabilidade da frequência cardíaca. Rev Port Cardiol. 1995; 14 (3): 241-62.

16. Grossman P, Wilhelm FH, Spoerle M. Respiratory sinus arrhythmia, cardiac vagal control and daily activity. Am J Physiol. 2004; 287: H728-H734.

17. Shin K, Minamitani H, Onishi S, Yamazaki H, Lee M. Autonomic difference between athletes and nonathletes: spectral analysis approach. Med Science Sports Exerc. 1997; 29: 1482-90.

18. Smith KR. Exercise can help control body changes during menopause. Health Care Food Nutr Focus. 2005; 22(10):10-2.

19. Katona PG, McLean M, Dighton DH, Guz A. Sympathetic and parasympathetic cardiac control in athletes and nonathletes at rest. J Appl Physiol. 1982, 89 $1652-7$
20. Akselrod S, Gordon D, Madwed JB, Snidman NC, Shannon DC, Cohen RI. Hemodynamic regulation: investigation by spectral analysis. Am J Physiol. 1985; 249: H867-H875.

21. Saul JP, Rea RF, Eckberg DL, Berger RD, Cohen RJ. Heart rate and muscle sympathetic nerve variability during reflex changes of autonomic activity. Am J Physiol. 1990; 258: H713-H721.

22. Sakabe DI, Catai AM, Neves VFC, Oliveira L, Silva de Sá MF, Azevedo GD, et al. Análise da modulação autonômica do coração durante condições de repouso em homens de meia-idade e mulheres pós-menopausa. Rev Bras Fisioter. 2004; 8 (1): 89-95.

23. Kimura T, Matsumoto T, Akiyoshi M, Owa Y, Miyasaka N, Aso T, Moritani T. Body fat and body lipids in postmenopausal women are related to resting autonomic nervous system activity. Eur J Appl Physiol. 2006; 97(5):542-7.

24. Malliani A, Pagani M, Lombardi F, Cerutti S. Cardiovascular neural regulation explored in the frequency domain. Circulation. 1991; 84: 1482-92.

25. Brockbank CL, Chaterjee F, Bruce SA, Woledge RC. Heart rate and its variability change after the menopause. Exp Physiol. 2000; 85 (3): 327-30.

26. Pines A, Fisman EZ, Drory Y, Levo Y, Shemesh J, Benari E, et al. Menopauseinduced changes in doppler-derived parameters of aortic flow in healthy women. Am J Cardiol. 1992; 69: 1104-6.

27. Schillaci G, Verdecchia P, Borgioni C, Ciucci A, Porcellati C. Early cardiac changes after the menopause. Hypertension. 1998; 32: 764-9.

28. Myslivecek PR, Broen CA, Wolfe LA. Effects of physical conditioning on cardiac autonomic function in healthy middle-aged women. Can J Appl Physiol. 2002; 27 (1): 1-18

29. Perini E, Fisher N, Veicsteinas A, Pendergast DR. Aerobic training and cardiovascular responses at rest and during exercise in older men and women. Med Sci Sports Exerc. 2002; 34 (4): 700-8.

30. Gregoire J, Tuck S, Yamamoto Y, Hughson RL. Heart rate variability at rest and exercise: influence of age, gender and physical training. Can J Appl Physiol. 1996; 21 (6): 455-70.

31. Tulppo MP, Timo H, Mäkikallio TH, Seppänen T, Laukkanen RT, Huikuri HV. Vagal modulation of heart rate during exercise: effects of age and physical fitness. Am J Physiol. 1998; 274 (Pt 2 2): H424-H429.

32. Carels RA, Darby LA, Cacciapaglia HM, Douglass OM. Reducing cardiovascular risk factors in postmenopausal women through a lifestyle change intervention. J Womens Health (Larchmt). 2004; 13 (4): 412-26.

33. Jurca R, Church TS, Morss GM, Jordan AN, Earnest CP. Eight weeks of moderate-intensity exercise training increases heart rate variability in sedentary postmenopausal women. Am Heart J. 2004; 147 (5): 8-15. 